\title{
Compatibility measure and penalized contact resolution for incompatible interfaces
}

\author{
Guillaume Vermot des Roches 1,2 _ Etienne Balmes 1,3 \\ Hachmi Ben Dhia ${ }^{2}$ - Rémi Lemaire ${ }^{4}$ - Thierry Pasquet ${ }^{4}$
}

1) SDTools, 44, Rue Vergniaud, F-75013, Paris

\{vermot,balmes\}@sdtools.com

2) Ecole Centrale Paris, CNRS, Laboratoire MSS-MAT

Grande Voie des Vignes

F-92295, Châtenay-Malabry

hachmi.ben-dhia@ecp.fr

3) Arts et Metiers ParisTech, CNRS, Laboratoire LMSP

151, Boulevard de l'hôpital, F-75013, Paris

4) Robert Bosch (France) SAS, Chassis System Brakes

126, Rue de Stalingrad, F-93700, Drancy

\{remi.lemaire, thierry.pasquet\}@fr.bosch.com

ABSTRACT. Handling of large industrial mechanical assemblies implies structure interactions commonly modeled with contact formulations. In cases where component interfaces are discretized using non conforming meshes, classical contact solutions have difficulties producing correct contact pressure fields. The method presented in this paper gives a relevant measure of interface compatibility and shows how it can be exploited to obtain regular contact pressures or limit over-integration in the contact formulation.

RÉSUMÉ. La gestion d'assemblages mécaniques complexes nécessite souvent des modèles d'interaction de structure par une formulation en contact. Lorsque la discrétisation éléments finis des interfaces est non compatible, les résolutions classiques peuvent êtres non régulières. La méthode présentée dans ce papier donne une mesure pertinente de la compatibilité des interfaces et montre comment l'exploiter pour régulariser les pressions de contact ou limiter la sur-intégration dans la formulation du contact.

KEYWORDS: penalized contact, regularization, (1-H)-compatibility, non-conforming mesh.

MOTS-CLÉS : contact pénalisé, régularisation, (1-H)-compatibilité, maillages non compatibles.

DOI:10.3166/EJCM.19.317-328 C 2010 Lavoisier, Paris

EJCM - 19/2010. Giens 2009, pages 317 to 328 


\section{Introduction}

The constant improvement of computational power allows the implementation of numerical prototyping at every stage of the design cycle, thus reducing costs and development time. In particular, simulation of complex mechanical assemblies with the finite element method is now widespread in industry. Such models however imply the ability to handle components interactions of discretized interfaces at the system level.

Interaction between moving parts typically requires contact models which can be split in two main categories, using either Lagrange or penalized formulations. Lagrange formulations enforce strict non-penetration, while penalization authorizes a relative level of interface interpenetration through a pressure-gap relationship. Both models are an idealization of contact, indeed, the reality of contacting surfaces implies interpenetration seen at the microscopic level as the compression of contacting asperities. Non linear penalization formulations, which is the modeling context of this study, can then be seen as a characterization at the system level of the local contact behavior.

External modeling and model generation constraints usually found for industrial models (automatic mesh generation at the component level, mesh provided by contractors,... ) often lead to non-conforming meshes for the contacting interfaces. The definition of master/slave surfaces is also a question that is often with no clear answer, in particular with partially covering surfaces due to large displacements or the presence of holes.

The problem of implementing bilateral or contact coupling for non-conforming meshes has been widely studied in the finite element (Babuska, 1973; Bernadi et al., 1992; Kim, 2002) and component mode synthesis (Balmes, 1996; de Klerk et $a l ., 2008)$ literature. The strategy presented here is based on the notions of pseudo compatibility introduced in (Ben Dhia et al., 2003) extended to tridimensional meshed volumes and the need to use specific integration rules for interfaces (Ben Dhia, 2002).

Section 2 illustrates the problematic and proposes a measure of non conforming interface compatibility which can be taken as a quality indicator. The measure is directly exploited in Section 3 to regularize contact solutions by describing interface fields as a linear combination of quasi-compatible shapes, through either primal or dual formulations. Section 4 eventually provides applications to an academic model showing large mesh refinement differences and to an industrial pad/disc model from Bosch, showing large non compatible interfaces. 


\section{A measure of interface compatibility}

A compatibility measure is an indicator of the interface ability to transmit a field from one surface to the other. For a general non conforming interface, not all field distributions can be equally represented by both surfaces. A measure of compatibility thus needs to evaluate differences between the representations of a given field on both surfaces.

\subsection{Illustration of the problem}

The usual finite element formulation of penalized contact can yield poor results in the case of non conforming interface meshes, especially when mesh sizes differ in contacting surfaces and partial element coverage is present - e.g. due to holes or global motion. The academic illustration presented in Figure 1 is a contact between two cubes in vertical translation. The lower cube is clamped at his base, drilled and finely meshed. The upper cube is plain, coarsely meshed and a pressure is applied to its top surface.
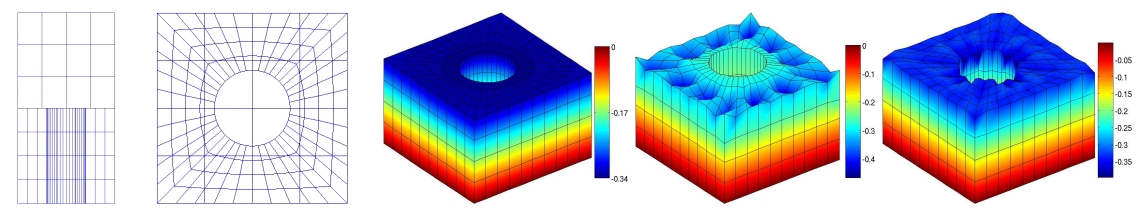

Figure 1. Example of incompatible mesh not covering. Reference solution is plotted in the middle, against the 1 (right) and 4 (far right) point rule integration solution using the upper cube as master

Figure 1 presents displacement results obtained for different formulations. Using the coarse upper cube as the master means that a lot of slave nodes are not matched (or not seen by any master contact point). The richer shapes of $\Gamma_{2}$ cannot be represented on $\Gamma_{1}$ and are therefore not seen nor constrained by the contact formulation on $\Gamma_{1}$. This pattern is close to hourglass modes observed for under-integrated elements.

Introducing very rich integration avoids oscillations but can significantly augment computational costs and leads to excessive stiffness, or locking, when zero gap is enforced strictly (Ben Dhia et al., 2003). The following sections will introduce an energy function for the gap and show how this can be used to quantify and possibly relax compatibility of displacements on the interfaces. 


\subsection{Definition of gap energy}

Contact between two solids $\Omega_{1}$ and $\Omega_{2}$, is defined between two surfaces $\Gamma_{1}$ and $\Gamma_{2}$. The compatibility is computed on the subset of effective contact $\Gamma$ of $\Gamma_{1} \times \Gamma_{2}$, as shown Figure 2.

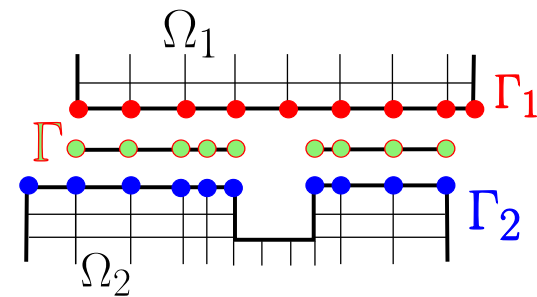

Figure 2. Definition of a domain $\Gamma$ for the compatibility computation

Any contact formulation needs to evaluate the gap field between two surfaces which is defined for a displacement $u\left(q_{1}\right)$ of $\Gamma_{1}$ and $u\left(q_{2}\right)$ of $\Gamma_{2}$, where $q_{1}$ (resp. $q_{2}$ ) is the discretized displacement on $\Gamma_{1}\left(\right.$ resp. $\left.\Gamma_{2}\right)$ as

$$
\left\{g\left(u(q 1), u\left(q_{2}\right)\right)\right\}=\left\{u\left(q_{1}\right)-u\left(q_{2}\right)\right\}^{T}\{n\}
$$

The first step is to define a scalar product on $\Gamma_{1} \times \Gamma_{2}$ that is a norm for the gap. The strain energy of a penalized contact with a uniform contact stiffness density $k$ is chosen here. For a displacement $u$, this energy is given by

$$
E_{p}=\int_{\Gamma} k g\left(u\left(q_{1}\right), u\left(q_{2}\right)\right)^{2} d S
$$

This scalar product must be approximated as precisely as possible (Ben Dhia, 2002). The computation strategy chosen here is an automated Delaunay triangulation over the subset $\Gamma$ of $\Gamma_{1} \times \Gamma_{2}$ in effective contact. The scalar product is computed by numerical quadrature from the mesh of $\Gamma$

$$
E_{p}=\sum_{x_{i}} k w_{i} J\left(x_{i}\right) g_{i}\left(u_{i}\left(q_{1}\right), u_{i}\left(q_{2}\right)\right)^{2}
$$

In practice, the gap is computed on $N_{\Gamma}$ integration points (Gauss) and linearly linked to the normal displacement observation matrices $\left[C_{N}^{1}\right]_{N_{\Gamma} \times N_{\Gamma_{1}}}\left(\right.$ resp. $\left.\left[C_{N}^{2}\right]_{N_{\Gamma} \times N_{\Gamma_{2}}}\right)$ of $\Gamma_{1}$ (resp. $\Gamma_{2}$ ) on $\Gamma$. This yields the discretized gap formulation

$$
\{g\}_{i}=\left[C_{N_{i}}^{1}\right]\left\{q_{1}\right\}-\left[C_{N_{i}}^{2}\right]\left\{q_{2}\right\}
$$

The contact strain energy is then of the form

$E_{p}=\left\{\begin{array}{l}q_{1} \\ q_{2}\end{array}\right\}^{T}\left[\begin{array}{ll}C_{N_{i}}^{1} & -C_{N_{i}}^{2}\end{array}\right]^{T}\left[\backslash k w_{i} J\left(x_{i}\right) \backslash\right]\left[\begin{array}{ll}C_{N_{i}}^{1} & -C_{N_{i}}^{2}\end{array}\right]\left\{\begin{array}{l}q_{1} \\ q_{2}\end{array}\right\}$ 
which defines the scalar product on $\Gamma$. The scalar product matrix is noted $[A]$ and is written by blocks

$$
E_{p}=\{q\}^{T}[A]\{q\}=\left\{\begin{array}{l}
q_{1} \\
q_{2}
\end{array}\right\}^{T}\left[\begin{array}{ll}
A_{11} & A_{21}^{T} \\
A_{21} & A_{22}
\end{array}\right]\left\{\begin{array}{l}
q_{1} \\
q_{2}
\end{array}\right\}
$$

\subsection{Robust compatibility computation}

In (Ben Dhia et al., 2003) the so-called $(1-\epsilon)$-compatibility is computed by considering the norm difference between a displacement $q_{1}$ of an interface $\Gamma_{1}$ and its projection to the facing interface $\pi_{2}^{1}\left\{q_{1}\right\} \in \Gamma_{2}$. The projection, defined as the vector of $\Gamma_{2}$ that minimizes the gap energy, verifies

$$
\left[A_{21}\right]\left\{q_{1}\right\}-\left[A_{22}\right] \pi_{2}^{1}\left\{q_{1}\right\}=0
$$

This suggests a formulation of the $(1-\epsilon)$-compatibility as a Rayleigh quotient, as

$$
C_{2}^{1}\left(\left\{q_{1}\right\}\right)^{2}=\frac{\left\|\pi_{2}^{1}\left\{q_{1}\right\}\right\|_{A_{22}}}{\left\|\left\{q_{1}\right\}\right\|_{A_{11}}}=\frac{\left\{q_{1}\right\}^{T}\left[A_{21}\right]^{T}\left[A_{22}\right]^{-1}\left[A_{21}\right]\left\{q_{1}\right\}}{\left\{q_{1}\right\}^{T}\left[A_{11}\right]\left\{q_{1}\right\}}
$$

Such formulation raises robustness issues as partial element covering tends to yield ill conditioning of $\left[A_{11}\right]$ and $\left[A_{22}\right]$. The formulation presented here computes the singular value decomposition (SVD) of $[A]$ (rather than $\left[A_{21}\right]$ suggested in (Ben Dhia et al., 2003)). As $[A]$ is symmetric,

$$
[A]=\left[\cdots\left\{\begin{array}{c}
u_{i}^{1} \\
u_{i}^{2}
\end{array}\right\} \cdots\right]\left[\backslash_{i \backslash}\right]\{U\}^{T}
$$

The SVD generates a displacement basis $\left\{u_{i}\right\}$ of the coupled interface with the level of gap energy generated $\sigma_{i}$. Low singular values thus characterize compatible interface displacements. The compatible displacement fields obtained are then known to be represented on both surfaces well enough - which will be quantified by a tolerance $\epsilon$. A new definition of interface compatibility is suggested as

$$
C_{2}^{1}(\epsilon)=\frac{\operatorname{card}\left\{i / \frac{\sigma_{i}}{\max _{i} \sigma_{i}} \leq \epsilon\right\}}{\min _{i=1,2}\left(\operatorname{card}\left(\Gamma_{i}\right)\right)}
$$

It is useful to note that the gap observation [4] can be performed with the full contact set. Unmatched contact DOF will generate zero terms on the diagonal of $[A]$. No conditioning issue affects the SVD computation which would output in that case a null (to numerical precision) singular value associated with shapes showing unmatched points displacements only. The description of the unmatched point displacements is then a full recombination of the finite element basis. The refinement of $\Gamma$ is nevertheless necessary for a good description of the non spurious contact points. 


\section{Using (1- $\epsilon)$ vector pairs to solve contact problems}

The compatibility measure through an SVD generates couples of quasi-compatible displacements on both sides of an interface. The following sections discuss their use for contact problems.

\subsection{Primal and dual formulation}

The primal formulation constrains the interface displacement to the subspace of quasi-compatible displacements. The model DOF are sorted to segregate the interface DOF $\left(q_{i 1}\right.$ and $\left.q_{i 2}\right)$ from the rest $\left(q_{c}\right)$, thus allowing the projection to the generalized quasi-compatible interface DOF $\left(q_{\epsilon 1}\right.$ and $\left.q_{\epsilon 2}\right)$. Regular contact force distributions are then a consequence of the imposed displacement regularity.

$$
\left\{\begin{array}{c}
q_{c} \\
q_{i 1} \\
q_{i 2}
\end{array}\right\}=\left[\begin{array}{ccc}
\mathcal{I} & 0 & 0 \\
0 & U_{\epsilon}^{1} & 0 \\
0 & 0 & U_{\epsilon}^{2}
\end{array}\right]\left\{\begin{array}{c}
q_{c} \\
q_{\epsilon 1} \\
q_{\epsilon 2}
\end{array}\right\}=\left[T_{\epsilon}\right]\left\{\begin{array}{c}
q_{c} \\
q_{\epsilon 1} \\
q_{\epsilon 2}
\end{array}\right\}
$$

The gap observation is consequently projected and can be written

$$
\{g\}=\left[C_{N i}^{1}\left[U_{\epsilon}^{1}\right]\right]\left\{q_{\epsilon 1}\right\}-\left[C_{N i}^{2}\left[U_{\epsilon}^{2}\right]\right]\left\{q_{\epsilon 2}\right\}
$$

Exact compatibility (zero gap on $\Gamma$ ) is known to generate locking issues, as discussed for example in (Balmes, 1996; Ben Dhia et al., 2003). Introducing quasicompatible displacements relaxes the constraint while avoiding large relative displacements.

In a dual formulation, the projection on the (1- $\epsilon)$-compatible displacement pairs of the contact forces is assumed to be zero. This clearly avoids local stress concentrations associated with locking but also leads to larger gaps. Dual formulations also have the advantage of preserving the use of physical displacement DOFs.

\subsection{Regularized gap function for non-linear contact laws}

In general penalized contact approaches, the load is a non linear function of the gap. Exponential functions are in particular used for brake squeal studies (Vermot des Roches et al., 2008). In a dual formulation, locking is avoided but the gap is no longer regular. It is thus proposed to use a regularized gap observation on $\Gamma$ to compute contact forces.

For a surface $\Gamma_{n}$ the regularized displacement is a linear combination of the trace on $\Gamma_{n}$ of the compatible vectors, $\left[U_{\epsilon}^{n}\right]$

$$
\left\{q_{n}^{\epsilon}\right\}_{N_{\Gamma_{n}} \times N_{\epsilon}}=\left[U_{\epsilon}^{n}\right]_{N_{\Gamma_{n}} \times N_{\epsilon}}\left\{\alpha_{n}\right\}_{N_{\epsilon}}
$$


where $\left\{\alpha_{n}\right\}$ is a solution of the minimization problem

$$
\left\{\alpha_{n}\right\}=\min _{\{\alpha\}}\left\|\left[U_{\epsilon}^{n}\right]\{\alpha\}-\left\{q_{n}\right\}\right\|_{\mathcal{K}}
$$

This defines a pseudo-inverse, as the solution searched is the closest to $\left\{q_{n}\right\}$ possible, relatively to a norm $\mathcal{K}$. The choice of $\mathcal{K}$ is fully open. It cannot be a restriction of $[A]$ due to conditioning problems, but considering a norm in displacement or strain on $\Gamma_{n}$ seems meaningful. The identity was chosen here so that the modified gap observation is

$$
\{g\}_{i}=\left[C_{N i}^{1}\left[U_{\epsilon}^{1}\right]\left[U_{\epsilon}^{1}\right]^{+}\right]\left\{q_{1}\right\}-\left[C_{N i}^{2}\left[U_{\epsilon}^{2}\right]\left[U_{\epsilon}^{2}\right]^{+}\right]\left\{q_{2}\right\}
$$

Projection of both sides of the interface can be considered. In master/slave configurations, a slave surface only projection is sufficient to suppress the observation of non quasi-compatible displacements.

\subsection{Opportunities for contact under-integration}

Obtaining proper quasi-compatible modes requires a good representation of the scalar product presented in Section 2.2. Relatively rich integration rules on both surfaces are therefore needed for these computation steps. However, the selection of quasi-compatible modes typically restricts the number of needed interface degrees of freedom significantly. The number of integration points needed for contact can consequently be decreased as information redundancy occurs. This is particularly interesting in vibration studies for which the contact surface is assumed constant during long time simulations, so that coarser rules will decrease computation time.

The proposed strategy is to use a fine integration rule to compute $[A]$ and $\left[U_{\epsilon}^{i}\right]$ defined in [8] and [9], then to switch to a coarser rule for the evaluation of the regularized gap [15] and resulting contact loads. Such under-integration could be optimized by the use of the Orthogonal Maximum Sequence method (Balmes, 2005) to create an optimal set of contact points associated to the quasi-compatible shapes to be observed.

\section{Illustrations}

The concepts of Sections 2 and 3 are applied to the academic model which features great mesh refinement differences, and to an industrial model featuring a relatively large interface. Regularization patterns are presented on the first case while under-integration strategies in the scope of an improvement of dynamical behavior are discussed on the latter model. 


\subsection{A drilled cube example}

This section illustrates the resolution method presented to the drilled cube example, using the upper cube as contact master, which yields poor results when no regularization is considered. The first step to the resolution is the creation of $\Gamma$, and the SVD computation of $[A]$, as plotted in Figure 3.

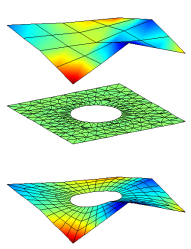

(a) Shape \#1, $\sigma=4.2 \cdot 10^{-18}$

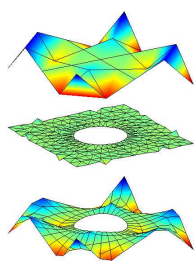

(b) Shape \#22,

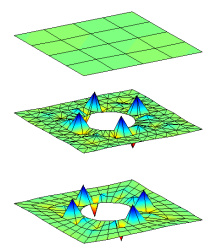

(c) Shape \#96, $\sigma=7.9 \cdot 10^{-2}$

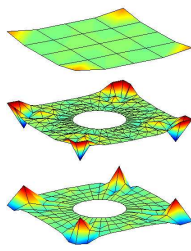

(d) Shape \#166,

Figure 3. Shapes resulting from the SVD of $[A]$ plotted on $\Gamma_{1}$ and $\Gamma_{2}$. The gap is computed and plotted on the Gauss points of $\Gamma$

The gap is computed on the Gauss integration points of $\Gamma$ for which a Delaunay triangulation is performed for visualization purposes. The compatible shapes shown in Figure $3 \mathrm{a}$ or $3 \mathrm{~b}$ correspond to very small gaps. The incompatible shapes in Figure $3 \mathrm{c}$ and $3 \mathrm{~d}$ show a high singular value ( $8 \%$ and $12 \%)$ and correspond to a large gap visible on $\Gamma$. In this problem, 25 quasi-compatible shapes at most exist since this is the number of DOF of the coarse interface.

To illustrate the regularized gap approach, the projected observation $\left[C_{N}^{1}\left[U_{\epsilon}^{1}\right]\left[U_{\epsilon}^{2}\right]^{+}\right]$is plotted and compared to $\left[C_{N}^{1}\right]$ in Figure 4 . The observation is the dual of the contact pressure as they are directly related by the pressure-gap contact law. The regularization method clearly distributes the gap observation to the contact point vicinity, instead of having a point-to-matched-point observation.

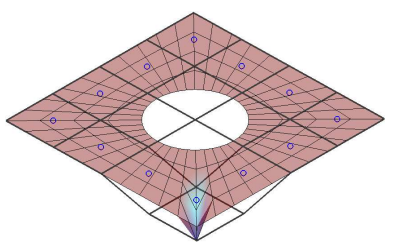

(a) Initial observation

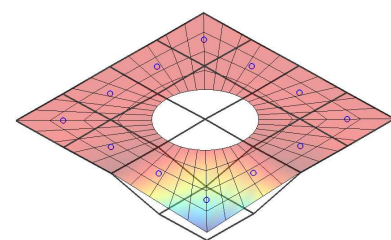

(b) Regularized observation

Figure 4. Observation for a given Gauss integration point (o) of the master surface 
Figure 5 plots gap results obtained for the center (one) point integration rule. The basic results are very poor as said earlier, and both primal and dual regularized solutions are satisfying.

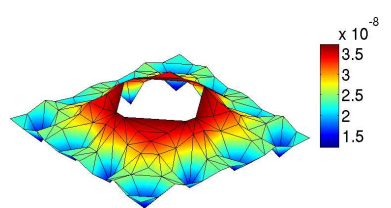

(a) Center rule

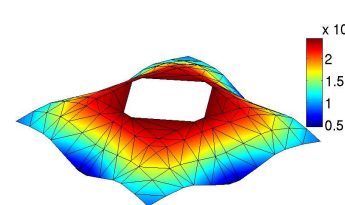

(b) Center Dual Reg.

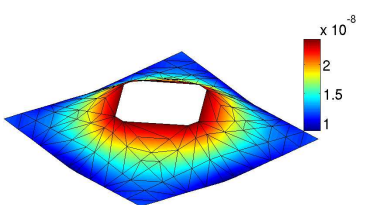

(c) Center Primal Reg.

Figure 5. Gap observations obtained using the center integration rule for contact, observed on the rich $3 \times 3$ rule Gauss points Delaunay triangulation, $\epsilon=10^{-5}$

Basic solutions provide poor contact fields, using a richer integration rule for that case is however an improvement. Indeed the approached contact area seen by the covered contact points increases by $25 \%$ when switching from the center point rule to the $2 \times 2$ points rule.

The primal resolution directly constraints displacements but can raise locking issues. No such pattern is observed for the cube and direct displacement restriction yields in this case better contact pressure fields. It is however costlier to implement as the interface projection would typically require DOF reordering prior to solve.

\subsection{Application to an industrial brake model}

State-of-the-art brake models used in this section are provided by Bosch. The system is meshed automatically by component using 10 node tetrahedron elements, resulting in non conforming interfaces. Figure 7 a shows the components retained, the disc and a pad with its backplate and lining. Pressure is applied using the trace of the caliper fingers on the backplate.

Static responses are properly handled here using a rich contact integration rule (even over-integration). For applications in dynamics, one seeks to compute small oscillations around a bilateral contact state. The measure of incompatibility introduced in the paper shows that although the mesh sizes are similar, compatibility is not very good. The combination of incompatible mesh and rich integration, then leads to locking issues which will be illustrated at the end of this section.

Applying the concepts presented in Section 2.2 leads to the compatible singular values and associated compatibility measure shown in Figure 6 . The first $10 \%$ of compatible shapes are free movements for the uncovered nodes of the partially covered elements. A threshold $\epsilon$ value is seen before more quasi-compatible shapes are found. 
More than $70 \%$ of shapes are above a $\epsilon=10^{-4}$ threshold. Thus although mesh sizes are similar, the compatibility is low. Enforcing strict displacement equality is thus expected to induce locking.

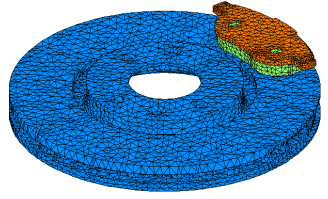

(a)

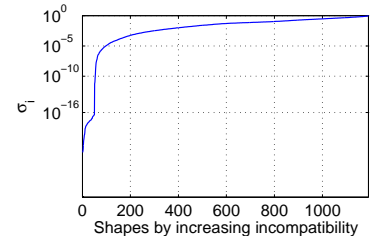

(c)

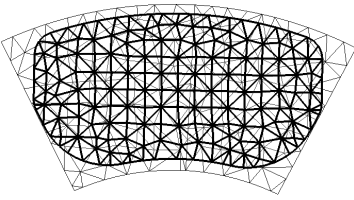

(b)

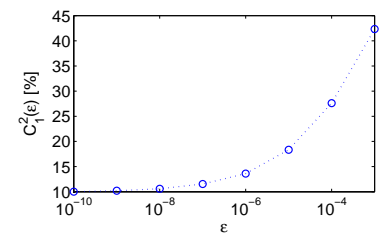

(d)

Figure 6. (a): Global view of the pad/disc model - (b) Superimposed pad/disc interface- $(c)$ : Singular values of $[A]-(d)$ : $(1-\epsilon)$-compatibility as function of $\epsilon$

Sample shapes resulting from the SVD are plotted in Figure 7. Shapes are plotted on $\Gamma_{1}$ and $\Gamma_{2}$ which are the upper and lower surfaces. The gap between both surfaces displacement is plotted on $\Gamma$ shown at the intermediate height.

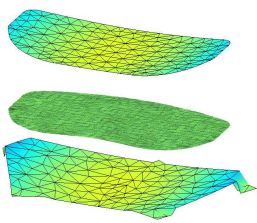

(a) Shape \#52, $\sigma=1.1 .10^{-1}$

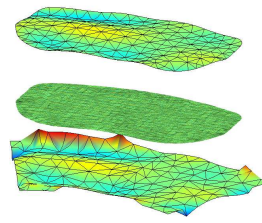

(b) Shape \#73, $\sigma=1 \cdot 1 \cdot 10^{-6}$

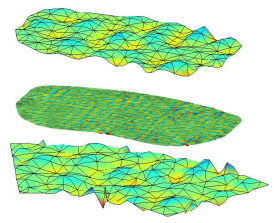

(c) Shape \#150, $\sigma=1.2 .10^{-4}$

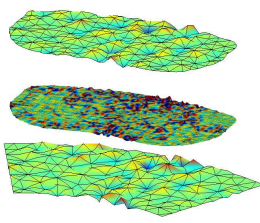

(d) Shape \#217, $\sigma=8.2 .10^{-4}$

Figure 7. Sample quasi-compatible shapes. $\Gamma$ is represented as a Delaunay triangulation of its Gauss points

The resolution of a contact problem with an exponential law is here satisfying using a rich integration rule ( 6 points for a 6 nodes triangle), but requires 1824 contact points. A center point integration rule uses only 394 points but results in strong contact irregularities. Indeed, the finite element formulation of a 6 node triangle allows the center point to move without a node movement. Further work on the reduction of the 
number of contact points will thus need to address the relation between the localization of contact points and the inherently non-local nature of $(1-\epsilon)$ compatible deformations.

As well documented in the literature, over-integration is a good solution to solve static contact problems. In dynamics, enforcing permanent contact exactly for incompatible meshes leads to locking. As an illustration, Figure 8 shows a transfer function from the vertical displacements of a disc point to a pad point. Modes are computed enforcing perfect bilateral contact (no gap) of the contact interface through either the basic contact normal displacement observation or the modified one (Equation [15]), using $\epsilon=10^{-3}$.

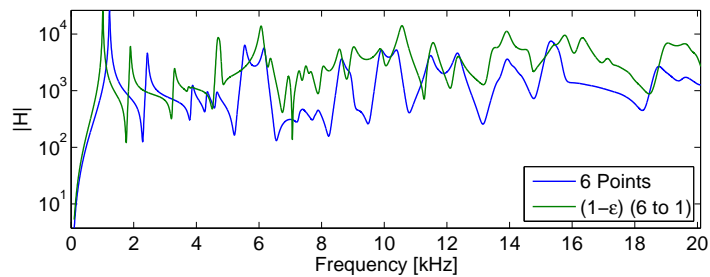

(a)

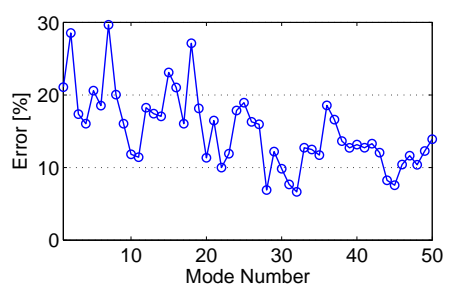

(b)

Figure 8. (a) Dynamic behavior differences using a 6 point integration rule, or the regularization strategy based on a 1 center point rule $\left(\epsilon=10^{-3}, 42 \%\right.$ of shapes kept). (b) Frequency differences observed

The over-integrated solution shows positive frequency shifts from 6 to $30 \%$, corresponding to resonance shifts visible even at low frequencies, and a clear stiffening (locking) of the structure. The application of interest being brake squeal simulations (Vermot des Roches et al., 2008), the typical frequency range of interest goes up to $16 \mathrm{kHz}$. The great differences observed in this range must clearly be addressed.

\section{Conclusion}

The article presented a general methodology to deal with field transmission in non conforming interface meshes. A scalar product on the interface is used to define compatible motion. Using a very rich integration for the evaluation of the gap energy, avoids stress concentrations associated with integration points in coarse meshes and is applicable to partially covered interfaces and cases where no obvious slave/master strategy exists.

Using rich integration, the contact must be verified at a high number of points so that only strictly compatible deformations of the interface are allowed. This leads to an over stiffening of the model, known as locking, which is particularly visible for applications in dynamics where the loads transmitted in the interface are not as smooth as in contact problems. 
A singular value decomposition of the gap energy operator generates pairs of (1- $\epsilon$ )-compatible deformations which handles difficult cases of very partial overlap. The relation between total number of DOF and number of $(1-\epsilon)$-compatible vectors is a direct numerical indicator of mesh compatibility. The industrial application shown illustrates the fact that meshes with comparable element sizes can have relatively low compatibility.

(1- $\epsilon$ )-compatible vector pairs can be used to avoid inappropriate gaps in a primal method that only allows compatible motion or to compute a regular pressure field by introducing a regularized gap function (dual method). An academic example illustrated how this is applicable to pathological cases such as gross under-integration which can be associated with the selection of a coarse master mesh. Another use might be the introduction of a coarse contact rule for applications in long transient simulations.

The need to relax continuity to avoid locking was finally illustrated for a real example. This leads to correct coupled behavior while avoiding pathologies of underintegration. It clearly appears that locking is particularly important for contact between solids. And that soft contact law, by opposition to Langrangian formulations or penalty approaches with stiff contact, will be less sensitive to the problem. The trade-off between the local nature of gap evaluations and the global nature of $(1-\epsilon)$ compatible deformations still needs further discussion.

\section{References}

Babuska I., “ The finite element method with Lagrange multiplier”, Numer.Math., vol. 20, p. $179-192,1973$

Balmes E., " Use of generalized interface degrees of freedom in component mode synthesis", International Modal Analysis Conference, p. 204-210, 1996.

Balmes E., “ Orthogonal Maximum Sequence Sensor Placements Algorithms for modal tests, expansion and visibility.", IMAC, January, 2005.

Ben Dhia H., Balmes E., “ Mesure de compatibilité et application aux problèmes de sousstructuration", Colloque National en Calcul des Structures, Giens, 2003.

Ben Dhia H.and Zarroug M., " Hybrid frictional contact particles-in elements”, Revue Européenne des Eléments Finis, vol. 11, n²-3-4, p. 417-430, 2002.

Bernadi C., Maday Y., Patera A., " Domain decomposition by the mortar element method", Laboratoire d'analyse numérique, Paris VI Univ., Report 92013, 1992.

de Klerk D., Rixen D., Voormeeren S. N., " General Framework for Dynamic Substructuring : History, Review and Classification of Techniques", AIAA Journal, vol. 46, n 5, p. 1169 1181,2008

Kim H.-G., “ Interface Element Method (IEM) for a partitioned system with non-matching interfaces", Comput. Methods Appl. Mech. Engrg., vol. 1, n 191, p. 3165-3194, 2002.

Vermot des Roches G., Balmes E., “ Time simulation of squeal phenomena in realistic brake models", Proceedings of the International Conference on Advanced Acoustics and Vibration Engineering (ISMA), p. 3007-3019, 2008. 